\title{
Expression of the SOCS family in human chronic wound tissues: Potential implications for SOCS in chronic wound healing
}

\author{
YI FENG ${ }^{1}$, ANDREW J. SANDERS ${ }^{1}$, FIONA RUGE ${ }^{1,2}$, CERI-ANN MORRIS $^{2}$, \\ KEITH G. HARDING ${ }^{2}$ and WEN G. JIANG ${ }^{1}$ \\ ${ }^{1}$ Cardiff China Medical Research Collaborative and ${ }^{2}$ Wound Healing Research Unit, \\ Cardiff University School of Medicine, Cardiff University, Cardiff CF14 4XN, UK
}

Received April 12, 2016; Accepted August 2, 2016

DOI: $10.3892 /$ ijmm.2016.2733

\begin{abstract}
Cytokines play important roles in the wound healing process through various signalling pathways. The JAK-STAT pathway is utilised by most cytokines for signal transduction and is regulated by a variety of molecules, including suppressor of cytokine signalling (SOCS) proteins. SOCS are associated with inflammatory diseases and have an impact on cytokines, growth factors and key cell types involved in the wound-healing process. SOCS, a negative regulator of cytokine signalling, may hold the potential to regulate cytokine-induced signalling in the chronic wound-healing process. Wound edge tissues were collected from chronic venous leg ulcer patients and classified as nonhealing and healing wounds. The expression pattern of seven SOCSs members, at the transcript and protein level, were examined in these tissues using qPCR and immunohistochemistry. Significantly higher levels of SOCS3 $(\mathrm{P}=0.0284)$ and SOCS4 $(\mathrm{P}=0.0376)$ in non-healing chronic wounds compared to the healing/healed chronic wounds were observed at the transcript level. Relocalisation of SOCS3 protein in the nonhealing wound environment was evident in the investigated chronic biopsies. Thus, the results show that the expression of SOCS transcript indicated that SOCS members may act as a prognostic biomarker of chronic wounds.
\end{abstract}

\section{Introduction}

The area of chronic wound healing has drawn much attention from many healthcare professionals due to the high cost of healthcare resources for patients seeking appropriate treatment, as well as the complexity of its underlying mechanism. It has been suggested that a reduction in tissue growth factors,

Correspondence to: Professor Wen G. Jiang, Cardiff China Medical Research Collaborative, Cardiff University School of Medicine, Henry Wellcome Building, Heath Park, Cardiff CF14 4XN, UK Email: jiangw@cf.ac.uk

Key words: suppressor of cytokine signalling, chronic wound, biopsies an imbalance between proteinases and their inhibitors, and the presence of senescent cells is of importance in chronic wounds (1). A variety of treatments, such as dressings, application of topical growth factors, autologous skin grafting and bioengineered skin equivalents have been applied to deal with certain types of chronic wounds in addition to the basic treatments (1). However, the specific mechanisms of each treament remain unclear and are under investigation. Therefore, more insight into the mechanisms responsible are required to gain a better understanding of the wound-healing process. Further clarification of this complex system may contribute to the emergence of a prognositc marker to predict the healing potential of chronic wounds, enhancing patient management.

Wound healing is a dynamic and interactive process that is immediately activated by the damage of skin upon injury. Primary targets for the treatment of wounds are reducing the healing time and generating an aesthetical scar or scar-free tissue without comprimising any normal function. The process of wound healing consists of three overlapping orchestrated stages, known as inflammation, proliferation, and re-epithelialization and tissue remodelling (2). These complex processes are regulated by numerous signalling pathways involving several cell types including keratinocytes, macrophages, fibroblasts and endothelial cells (3). A vast array of cytokines and growth factors play key roles in the process of wound healing through the initiation of a variety of signalling cascades. Disruption of these tightly regulated processes can lead to a variety of pathological conditions. The Janus kinase/signal transducer and activator of the transcription (JAK/STAT) pathway is a key pathway, responding to a wide variety of cytokines and growth factors. Any mutation that holds the potential to compromise the regular function of JAK/STAT pathway may affect cytokine stimulated signalling (4). By contrast, loss of regulation to this signalling pathway may cause inflammatory disease, erythrocytosis, gigantism and leukaemia (5). Therefore, the mechanism regulating appropriate JAK/STAT signalling is of great importance. This target can be achieved by so-called negative regulators, such as Src-homology 2 (SH2) containing phosphatase (SHP), protein inhibitors against STATs (PIAS) and suppressor of cytokine signalling (SOCS), which all comprise specific SH2 domain in their structure (6). However, compared to the other two cytokine signalling inhibitors which are constitutively expressed, SOCS is the only cytokine 
inducible inhibitor that may hold the potential to be utilised as a regulator for the dysregulation of cell metabolism.

SOCS protein was origninally identified in 1997 independently by three different groups as SOCS1, the JAK-binding protein (JAB) and the STAT-induced STAT inhibitor (SSI) (7-9). It was subsequently confirmed that all three belong to the SOCS family, which consists of eight members [SOCS1 to 7 and cytokine inducible SH2-containing protein (CIS)] in mammals (10). Generally, there are three major domains contributing to the function of SOCS, identified as a central conserved $\mathrm{SH} 2$ domain, a N-terminal domain of variable length and divergent sequence, and a carboxy-terminal, 40-amino-acid module known as SOCS box (11). Some of the structurally related eight SOCS family members are capable of attenuating cytokine signalling by blocking JAK tyrosine kinase activity, competing for a docking site with STAT protein and/or binding to the respective target proteins for subsequent proteosomal degradation (11). Of the eight SOCS family members, CIS/SOCS1 to SOCS3 are the most well characterised, whereas, the precise function of the remaining four SOCS proteins, SOCS4 to 7, have yet to be elucidated due to their extensive expression and inconsistent protein targets (12). However, evidence from existing studies show that SOCS family members can be classified according to their target proteins. CIS/SOCS1 to SOCS3 regulate cytokine receptor signalling through the JAK-STAT pathway, whereas SOCS4 to 7 are associated with the regulation of growth factor receptor signalling (13-15).

The SOCS family members have been found to regulate a number of cytokines and growth factors that are essential in the wound-healing process (16). With the absence of SOCS3, interleukin-6 (IL-6), a proinflammaroty cytokine, acts as an immunosuppressive cytokine that reduces tumour necrosis factor (TNF) induced by lipopolysaccharides (LPSs) (17). Additionally, SOCS3 selectively inhibits the activation of STAT3 signalling by IL-6, but does not influence IL-10-activated STAT3 signalling due to inefficient binding to the IL-10 receptor. This potentially explains the opposing function of the proinflammatory cytokine IL-6 and the anti-inflammatory cytokine IL-10, and indicates the regulatory role of SOCS3 to IL-6-induced STAT3 signalling, highlighting a potential role for SOCS3 in the treatment of IL-6-mediated inflammatory diseases (17). The expression of SOCS4 and 5 in HeLa cells is induced at the mRNA level following treatment with epidermal growth factor (EGF), which is frequently induced during the wound-healing process. In addition, either SOCS4 or 5 markedly reduces EGFR expression (13). This result suggests that SOCS4 and 5 may have an effect on EGF signalling through the regulation of EGFR, the respective receptor of EGF. SOCS5 is also capable of reducing the expression level of EGFR in a ligand- and c-Cbl-independent manner to enhance its proteasome degradation. In addition, $\mathrm{SH} 2$ and SB domains of SOCS5 are required for the regulation of EGFR, which enables SOCS5 to attenuate EGF-induced signalling by translocating EGFR to intracellular vesicles (13).

The present study examined the transcript expression profile of SOCS1 to 7 in a clinical cohort of healing and nonhealing chronic wounds. This expression was further examined in clinical tissue sections to demonstrate their expression pattern and localisation in these contrasting subtypes of chronic wounds.

\section{Materials and methods}

Tissue collection and processing. A clinical chronic wound tissue cohort was used in the present study. Tissues were collected from patients attending the University Hospital of Wales Wound Healing Clinic. Wound edge tissues were collected from chronic venous leg ulcer patients following ethics approval from a Local Committee (South East Wales Research Ethics Committee). Wound size was recorded at the time of initial biopsy and was re-measured after 3 months, during which wounds were treated as per best medical treatment. Wounds that had become reduced in size over this period were described as 'healing/healed' $(n=20)$ and those which remained static or grew in size were described as 'non-healing' $(n=51)$ chronic wounds. This cohort has been previously described (18).

Sample biopsies were snap frozen and stored at $-80^{\circ} \mathrm{C}$ before being placed in liquid nitrogen until required for processing and analysis. The samples were sectioned $(7 \mu \mathrm{m})$ on a cryostat (Leica Microsystems, Ltd., Milton Keynes, UK), for immunohistochemical (IHC) staining and multiple sections $(20 \mu \mathrm{m})$, were processed for the extraction of RNA, reverse transcription and transcript expression analysis using quantitative polymerase chain reaction (qPCR).

RNA extraction and reverse transcription. Multiple sections from the same patient sample biopsy were combined and homogenised in ice-cold TRIzol reagent (Sigma-Aldrich, Poole, UK) using a handheld homogeniser (Cole-Parmer, London, UK). RNA was subsequently extracted following the manufacturer's instructions. Following extraction, RNA was resuspended in diethyl pyrocarbonate (DEPC) $\mathrm{H}_{2} \mathrm{O}$ and quantified using a spectrophotometer (WPA UV 1101; Biotech Photometer, Cambridge, UK). The samples were then standardised prior to undertaking the reverse transcription reaction using an RT kit (Bio-Rad, Hemel Hemstead, UK) to generate cDNA.

$q P C R$. qPCR was undertaken to analyse transcript expression of the SOCS family members in the chronic healing and non-healing wound samples. This process has been previously described by our laboratory $(18,19)$. In brief, primers were designed for each of the SOCS family members using the Beacon Designer software (Bio-Rad, Hercules, CA, USA). A Z sequence was added to one of each of the primer pairs (ACTGAACCTGACCGTACA), to allow incorporation of the FAM tagged UniPrimer probe (Intergen, Inc., New York, NY, USA) and thus fluorescent detection. Expression of the target sequence was detected in conjunction with an internal standard used to generate a standard curve. The samples were amplified and detected on a StepOnePlus qPCR machine (Life Technologies, Paisley, UK), using qPCR mastermix (iQ supermix; Bio-Rad), with the specific forward primer $(10 \mathrm{pM})$, reverse primer containing the $\mathrm{Z}$ sequence (1 pM) and the FAM-tagged UniPrimer probe $(10 \mathrm{pM})$. SOCS family members were detected in conjunction with the cytokeratin 19 (CK19) housekeeping 
Table I. Primers used in the present study.

\begin{tabular}{lll}
\hline Primers & \multicolumn{1}{c}{ Forward } & \multicolumn{1}{c}{ Reverse } \\
\hline SOCS1 & 5'-GATGGTAGCACACAACCAG & 5'-ACTGAACCTGACCGTACAGAGGAGGAGGAGGAAGGTT \\
SOCS2 & 5'-GGATGGTACTGGGGAAGTAT & 5'-ACTGAACCTGACCGTACATGGGAGCTATCTCTAATCAA \\
SOCS3 & 5'-TCAAGACCTTCAGCTCCA & 5'-ACTGAACCTGACCGTACAGTCACTGCGCTCCAGTAG \\
SOCS4 & 5'-GGCAGTGTTTTCAATAAAG & 5'-ACTGAACCTGACCGTACAAGGTGGGAAAGGACACTTAT \\
SOCS5 & 5'-AGTCAAAGCCTCTCTTTTCC & 5'-ACTGAACCTGACCGTACACATTTGGGCTAAATCTGA \\
SOCS6 & 5'-CCTTACAGAGGAGCTGAAAA & 5'-ACTGAACCTGACCGTACACGAAAAGAAAAGAACCATC \\
SOCS7 & 5'-CAGGCCCTGAATTACCTC & 5'-ACTGAACCTGACCGTACAGAGGTTGCTGCTGCTGCT \\
CK19 & 5'-AGCCACTACTACACGACCAT & 5'-ACTGAACCTGACCGTACATCGATCTGCAGGACAATC
\end{tabular}

ACTGAACCTGACCGTACA represents the Z sequence. SOCS, suppressor of cytokine signalling; CK19, cytokeratin 19.

gene and values were subsequently normalised against this gene. Primer details are provided in Table I.

IHC staining. Primary antibodies: anti-SOCS1 (sc-9021), anti-SOCS2 (sc-9022), anti-SOCS3 (sc-9023), anti-SOCS4 (sc-68827), anti-SOCS5 (sc-100858), anti-SOCS6 (sc-5608) and anti-SOCS7 (sc-8291 and sc-137241) were purchased from Insight Biotechnology (Wembley, UK). All the antibodies were used at a final concentration of $2 \mu \mathrm{g} / \mathrm{ml}$.

IHC analysis was performed on a subset of chronic wound tissues that included healing/healed $(n=10)$ and non-healed $(\mathrm{n}=10)$ biopsies. Staining was performed using a standard avidin-biotin peroxidase technique. In brief, the frozen sections were fixed for $15 \mathrm{~min}$ in dried acetone (Thermo Fisher Scientific, Ltd., Loughborough, UK) and then air dried for $15 \mathrm{~min}$. The sections were washed three times in Tris-buffered saline (TBS) for 5 min each, followed by a 30 -min permeabilisation wash with $0.1 \%$ saponin/TBS (Sigma-Aldrich). Since this reaction is reversible, subsequent washes were performed with $0.1 \%$ saponin/TBS. The sections were then incubated with a blocking solution in a humidified box at room temperature $\left(20-22^{\circ} \mathrm{C}\right)$ for $1 \mathrm{~h}$. The blocking solution, which contained $0.1 \% \mathrm{BSA} / 0.1 \%$ saponin $/ 10 \%$ horse serum in TBS, was also used to dilute all subsequent reagents. Excess blocking solution was removed and the sections were incubated with the relevant, previously optimised, primary antibody for $1 \mathrm{~h}$. Following washing with $0.1 \%$ saponin/TBS, the sections were then incubated for 30 min with the relevant biotinylated secondary antibody (Vector Laboratories, Peterborough, UK), followed by a 30-min incubation with the avidin-biotin complex (ABC) reagent. The 3,3'-diaminobenzidine (DAB) substrate $(5 \mathrm{mg} / \mathrm{ml})$ was used to develop the final reaction product, the sections were rinsed in tap water, counterstained with Gill's hematoxylin (Vector Laboratories), and rehydrated through a series of graded alcohols, cleared in xylene (Thermo Fisher Scientific, Ltd.) and mounted in DPX (Merck Millipore, UK). Negative controls were performed where primary antibody was replaced with wash buffer.

Staining was visualised using a microscope, and the localisation and intensity of staining for each antibody was assessed by two independent investigators, at both the wound edge and an area distal to the wound edge. Intensity of staining was qualitatively graded as strong, moderate or negative.
Statistical analysis. Statistical analysis was undertaken using the SigmaPlot 11 statistical software package (Systat Software Inc., London, UK). Data were analysed using a twosample, two-tailed, t-test or a Mann-Whitney test depending on data normality. Values of $\mathrm{P}<0.05$ were regarded as statistically significant.

\section{Results}

Expression levels of seven SOCS family members in healing/healed and non-healing chronic wounds. The expression levels of seven SOCS family members were quantified in a cohort of venous leg ulcer patients with chronic wounds using qPCR (Fig. 1). The expression of each member of the SOCS family was obtained between healing/healed and non-healing chronic wounds. Compared to healing/healed chronic wounds, the non-healing chronic wound tissues had significantly higher levels of SOCS3 (P=0.0284) and SOCS4 $(\mathrm{P}=0.0376)$ transcripts. Similar to the expression levels of SOCS3 and 4, the enhanced expression levels of SOCS1, 2, 5 and 6 were observed in the non-healing chronic wounds compared to the healing/healed chronic wounds. However, the results did not show any significant differences $(\mathrm{P}>0.05)$. In contrast to the trend of expression levels in SOCS1 to 6, the expression level of SOCS7 was elevated in the healing/healed chronic wounds when compared to that of the non-healing chronic wounds, albeit the result was not statistically significant $(\mathrm{P}>0.05)$.

Protein expression levels of seven SOCS family members in chronic tissues

SOCS1 staining in chronic tissues. The IHC analysis revealed the majority of chronic wounds stained positively for SOCS1 protein (15/20 chronic wounds). Expression of SOCS1 was observed in 7/10 healing/healed chronic wounds and 8/10 non-healing chronic wounds, and therefore there were no distinct differences between the two groups. The majority of the positive biopsies expressed moderate levels of SOCS1, with the exception of one non-healing chronic wound where the expression was defined as strong (Fig. 2).

A comparison of SOCS1 expression at the wound edge to that observed distal to the wound (towards normal tissue) revealed that 6/20 biopsies had an increased expression of SOCS1 distal to the wound edge, although no difference was evident between 

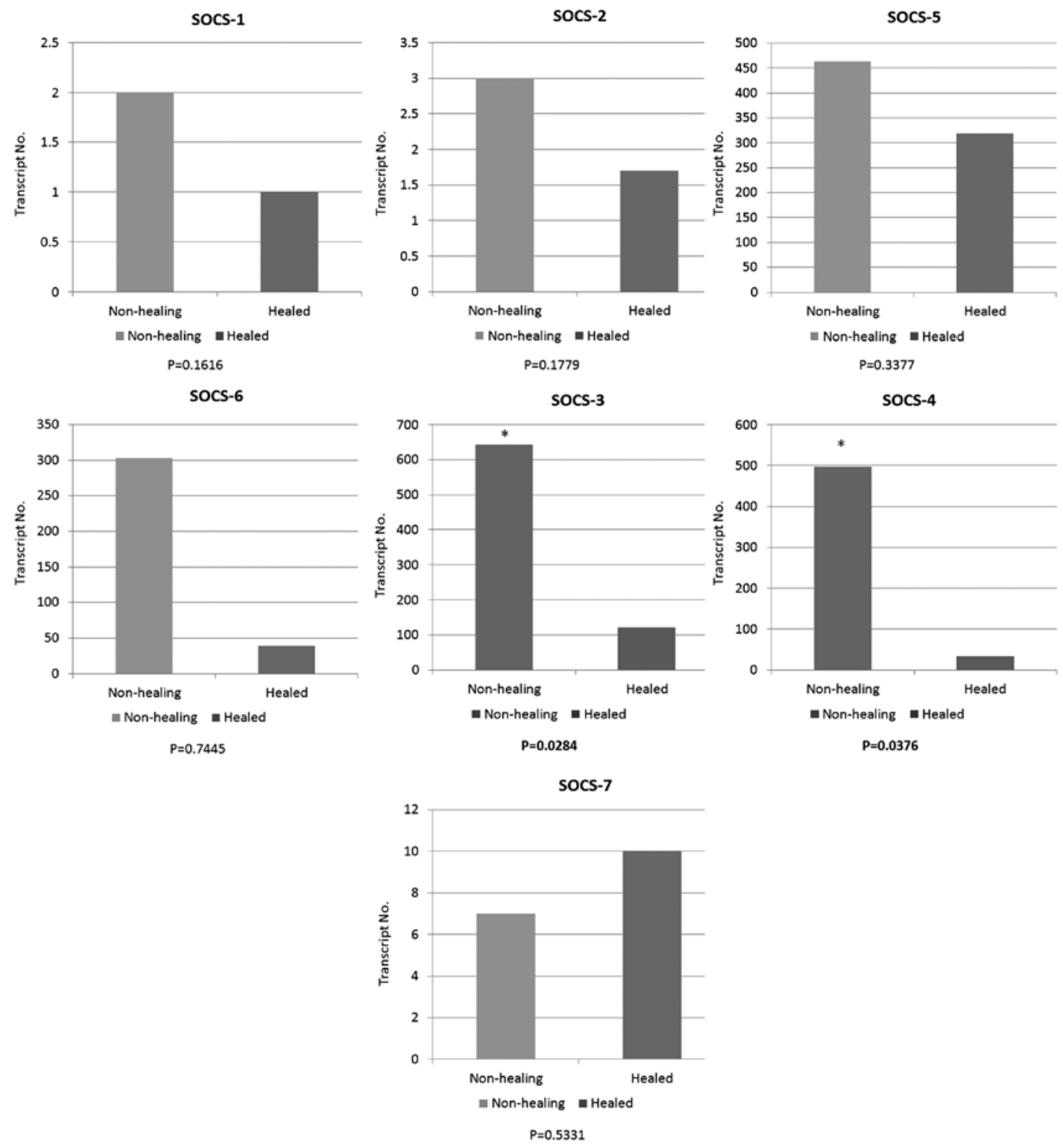

Figure 1. Transcript levels of suppressor of cytokine signalling 1 (SOCS1) (top panel left), SOCS2 (top panel middle), SOCS3 (second panel middle), SOCS4 (second panel right), SOCS5 (top panel right), SOCS6 (second panel left) and SOCS7 (bottom panel) in wound tissues. The expression of each SOCS family is normalised by the expression of cytokeratin 19 (CK19), ${ }^{*} \mathrm{P}<0.05$.

the healing/healed and non-healing groups (3/10 healing/healed and 3/10 non-healed). Generally, in the chronic wounds, diffuse cytoplasmic staining was observed in the mature keratinocytes of the epidermis, although increasing nuclear and cytoplasmic intensity in the basal layer distal to the wound edge was identified. Most blood vessels were weakly positive for SOCS1 in all the biopsies analysed. Positive staining was also observed in the dermal inflammatory infiltrate of some biopsies, but dual fluorescent staining was required to determine whether these were T-lymphocytes, macrophages, fibroblasts or other cells.

SOCS2 staining in chronic tissues. SOCS2-positive staining was found in 17/20 chronic wound biopsies studied, 8/10 healing/healed and 9/10 non-healing biopsies, respectively. The majority of these positively stained biopsies exhibited SOCS2 expression of moderate intensity, albeit $3 / 20$ revealed stronger intensity $(2 / 10$ healing/healed, 1/10 non-healing) (Fig. 3). Three of the positively stained biopsies showed SOCS2 expression in the dermal cell infiltrate, while no expression was observed in the epidermis.

SOCS2 protein expression was uniform throughout the whole biopsy, with only four chronic wounds showing differences between the wound edge and distal to the wound (3/10 healing/healed, 1/10 non-healing). Within the healing/healed subset, two of these biopsies showed an increased expression distal to the wound edge, whereas the other subset demonstrated an increased SOCS2 in the dermal infiltrate directly below the leading migratory epidermis (which was negatively stained).

Expression of SOCS2 protein was generally observed as diffuse cytoplasmic in mature keratinocytes, or nuclear in the lower keratinocytes of the basal layer. Membranous staining was observed in the very mature keratinocytes of some biopsies.

SOCS3 staining in chronic tissues. Positive SOCS3 staining was observed in the non-healing chronic wounds (10/10), and in the majority of healing/healed chronic wounds (8/10). Most 

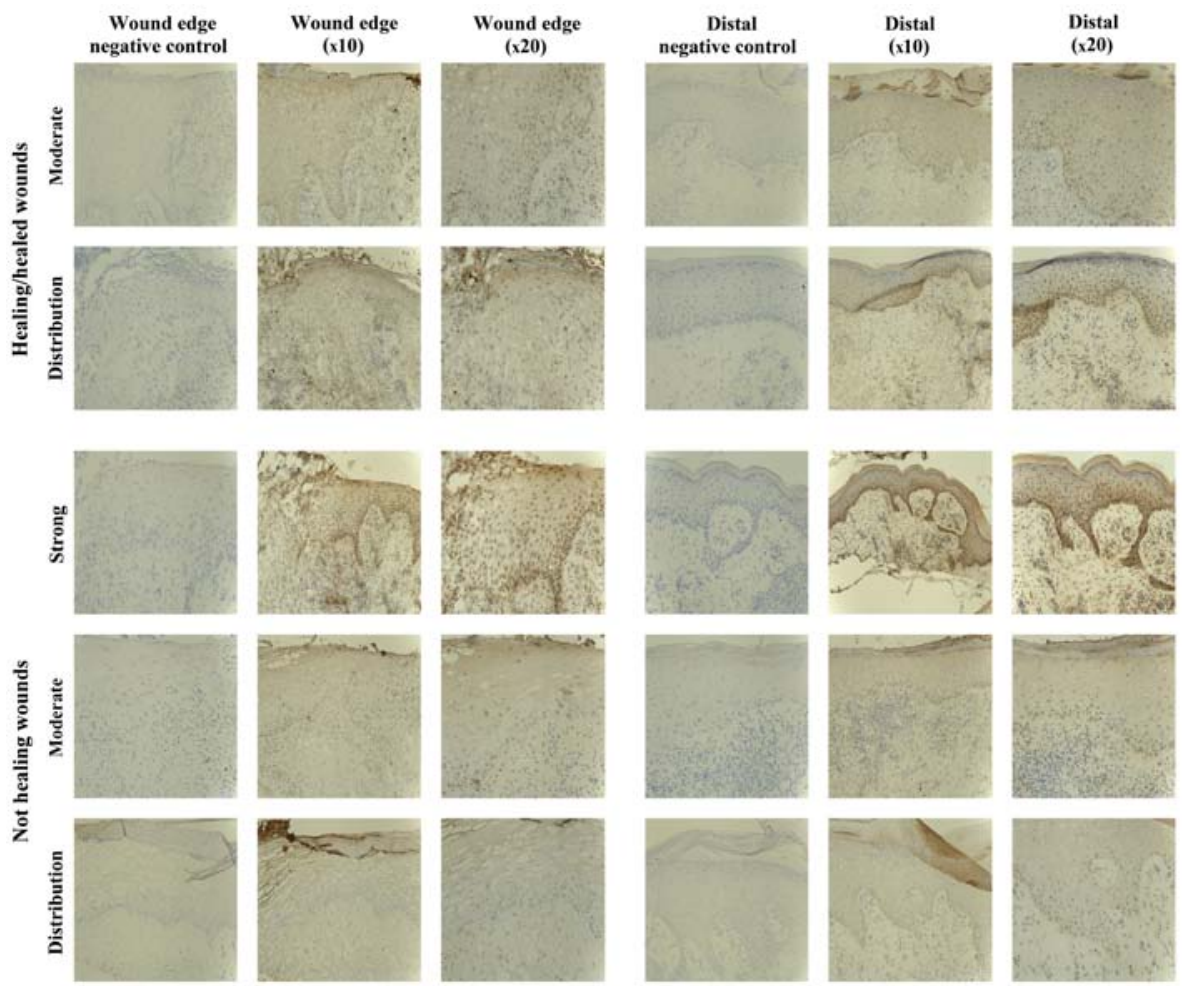

Figure 2. Immunohistochemical (IHC) evaluation of the suppressor of cytokine signalling 1 (SOCS1) protein in healing/healed wounds (top two panels) and non-healing wounds (bottom three panels). Each group shows representative images from collected biopsies, showing moderate SOCS1 staining and different protein distribution between wound edge and distal areas at two magnifications (x10 and x20). Representative images of non-healing sections demonstrate areas of strong and moderate staining as well as protein distribution (bottom panels).
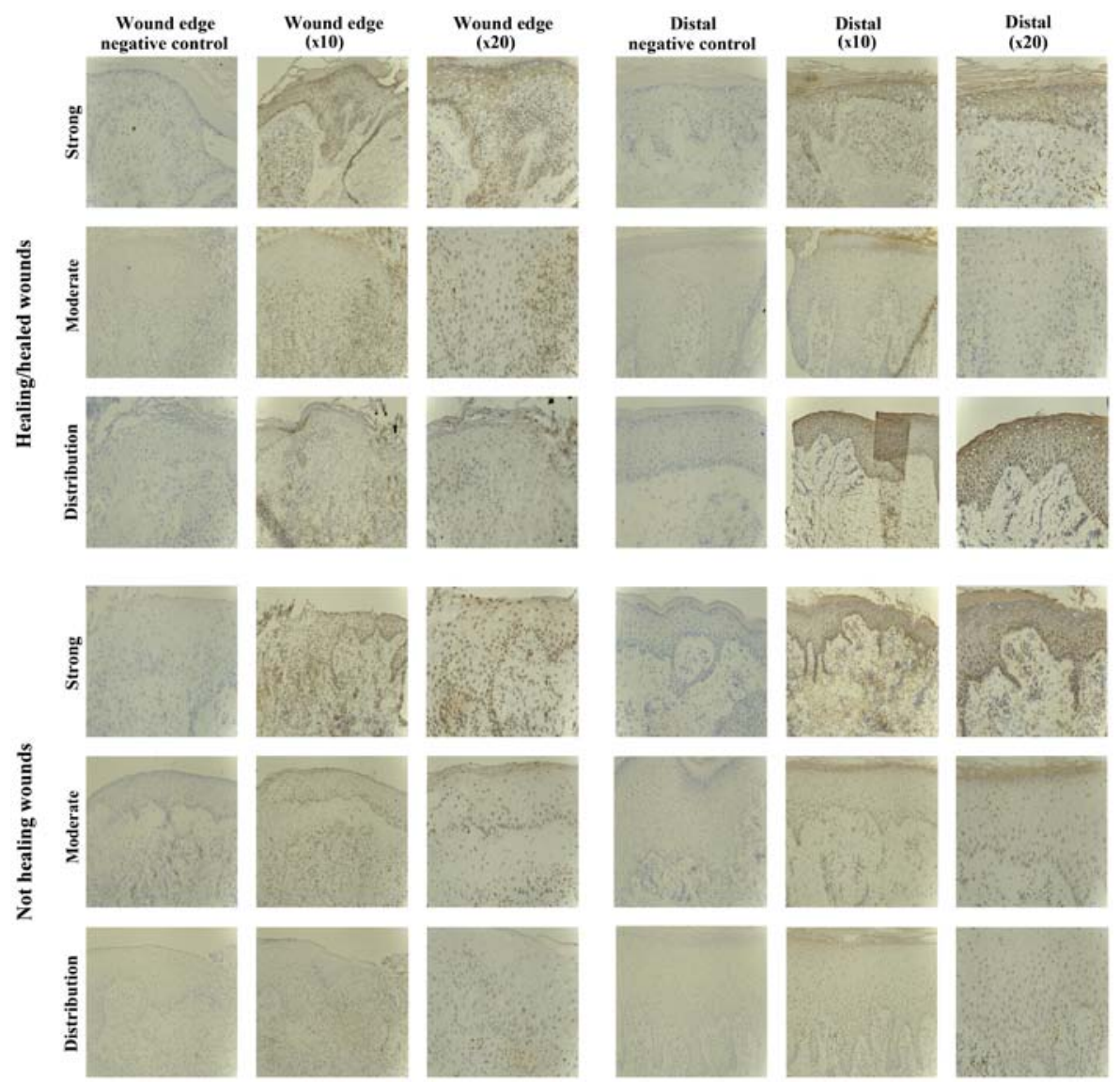

Figure 3. Immunohistochemical (IHC) evaluation of the suppressor of cytokine signalling 2 (SOCS2) protein in healing/healed wounds (top three panels) and non-healing wounds (bottom three panels). Each group shows representative images from the collected biopsies demonstrating strong staining, moderate staining and different protein distribution between wound edge and distal area at two magnifications (x10 and x20) within the sections. 

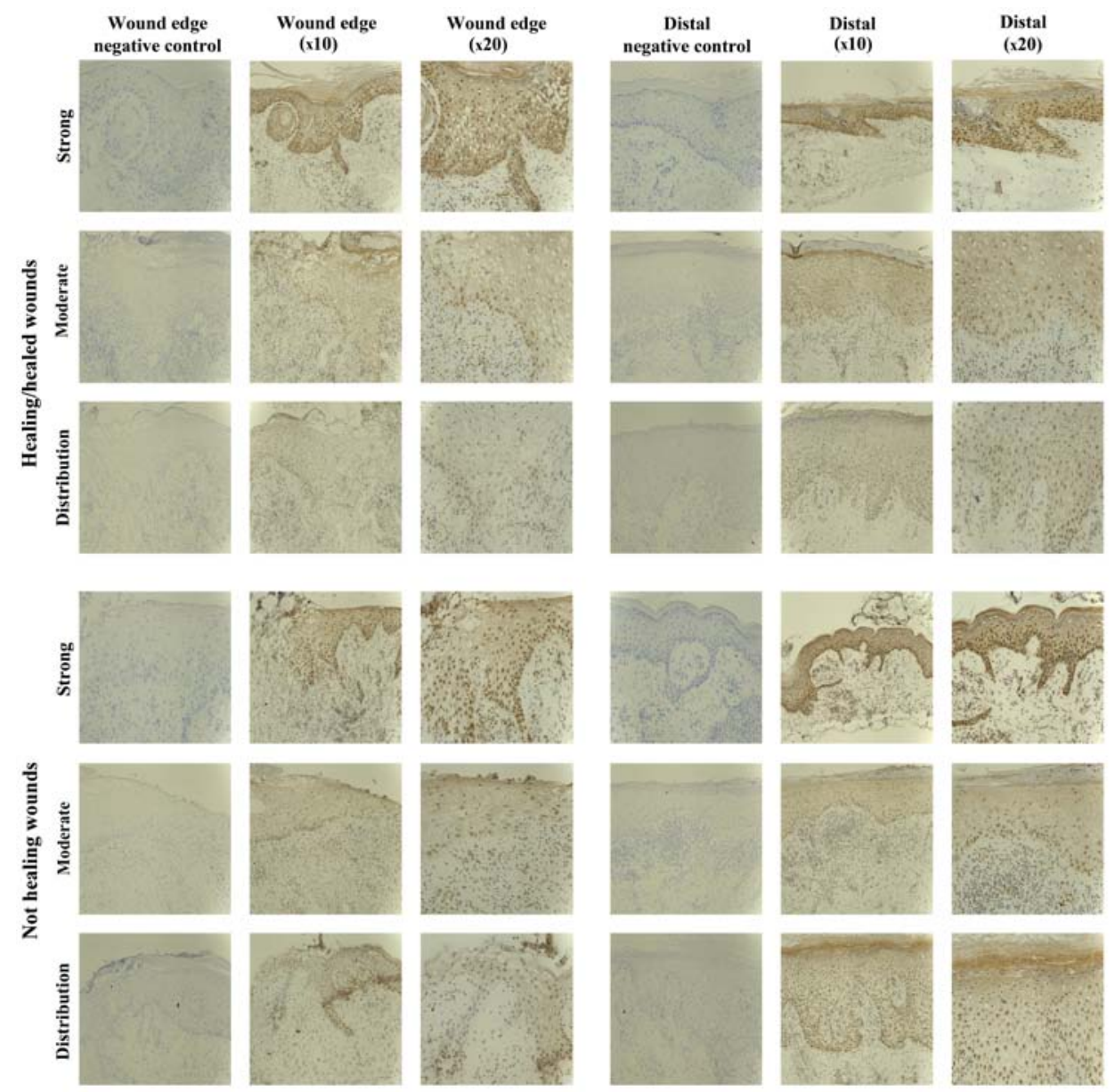

Figure 4. Immunohistochemical (IHC) evaluation of the suppressor of cytokine signalling 3 (SOCS3) protein in healing/healed wounds (top three panels) and non-healing wounds (bottom three panels). Each group shows representative images from the collected biopsies, demonstrating strong staining, moderate staining and different protein distribution between wound edge and distal area at two magnifications (x10 and x20) within the sections.

of the positively stained biopsies exhibited SOCS3 staining of moderate intensity (5/10 healing/healed, $8 / 10$ non-healing), but strong staining was observed in $3 / 10$ healing/healed and $2 / 10$ non-healing wounds (Fig. 4).

Five of the chronic wounds studied showed increased expression of SOCS3 protein in the epidermis distal to the wound edge, and interestingly the majority of these were non-healing chronic wounds $(1 / 10$ healing/healed, 4/10 non-healing), suggesting a trend of increased expression and relocalisation of SOCS3 in the non-healing wound environment. The remaining 15/20 chronic wounds studied had uniform epidermal staining throughout the biopsies.

SOCS3 expression was seen as diffuse cytoplasmic staining in the mature keratinocytes or nuclear in the lower basal and suprabasal layers, particularly distal to the wound edge. Many inflammatory cells within the dermis also expressed SOCS3, particularly below the leading wound edge (migratory epidermis) and around the blood vessels.

SOCS4 staining in chronic tissues. The SOCS4 protein expression was observed in almost all the chronic wound biopsies studied (18/20), and no distinct differences were evident between the two subsets $(9 / 10$ healing/healed, 9/10 non-healing). The majority of chronic wounds had uniform staining of moderate intensity throughout the biopsies $(16 / 20)$, and only two samples exhibited increased (strong) staining for SOCS4 (1/10 healing/healed, 1/10 non-healing) (Fig. 5).

Increased expression of SOCS4 distal to the wound edge was evident in 5/10 chronic wounds examined, and this was observed in the healing and non-healing wounds (3/10 healing/healed, 2/10 non-healing).

SOCS4 expression was mostly localised to the nucleus of the lower basal and suprabasal cells of the epidermis, but diffuse cytoplasmic staining was also observed in the very mature upper keratinocyte layers.

SOCS5 staining in chronic tissues. Staining of SOCS5 protein was generally less compared to that observed for the other SOCS proteins, but was obseved in almost all the chronic wounds studied (8/10 healing/healed, 8/10 non-healing) (Fig. 6). Uniform expression was observed in the two subsets, albeit 2/10 non-healing biopsies showed an increased SOCS5 expression distal to the wound edge. SOCS5 staining was mainly cytoplasmic, and observed in all the layers of the epidemis.

SOCS6 staining in chronic tissues. SOCS6 protein expression was observed in 12/20 chronic wounds, equally in the healing/healed (6/10) and non-healing chronic wounds (6/10). The IHC analysis revealed the staining to be of moderate intensity in most biopsies (5/10 healing/healed, 5/10 non-healing), 

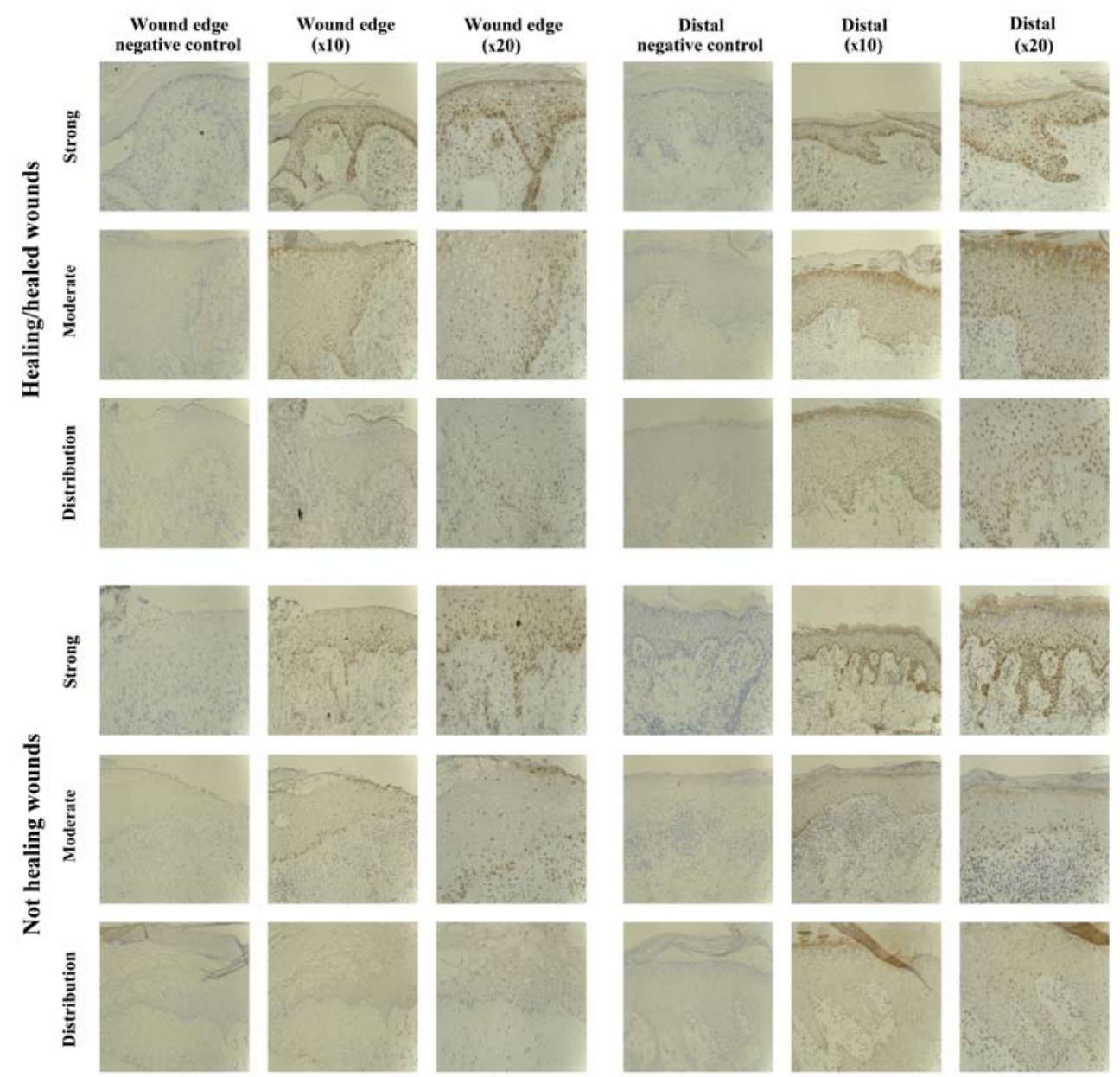

Figure 5. Immunohistochemical (IHC) evaluation of the suppressor of cytokine signalling 4 (SOCS4) protein in healing/healed wounds (top three panels) and non-healing wounds (bottom three panels). Each group shows representative images from the collected biopsies, which are strong staining, moderate staining and different protein distribution between wound edge and distal area at two magnifications (x10 and x20) within the sections.
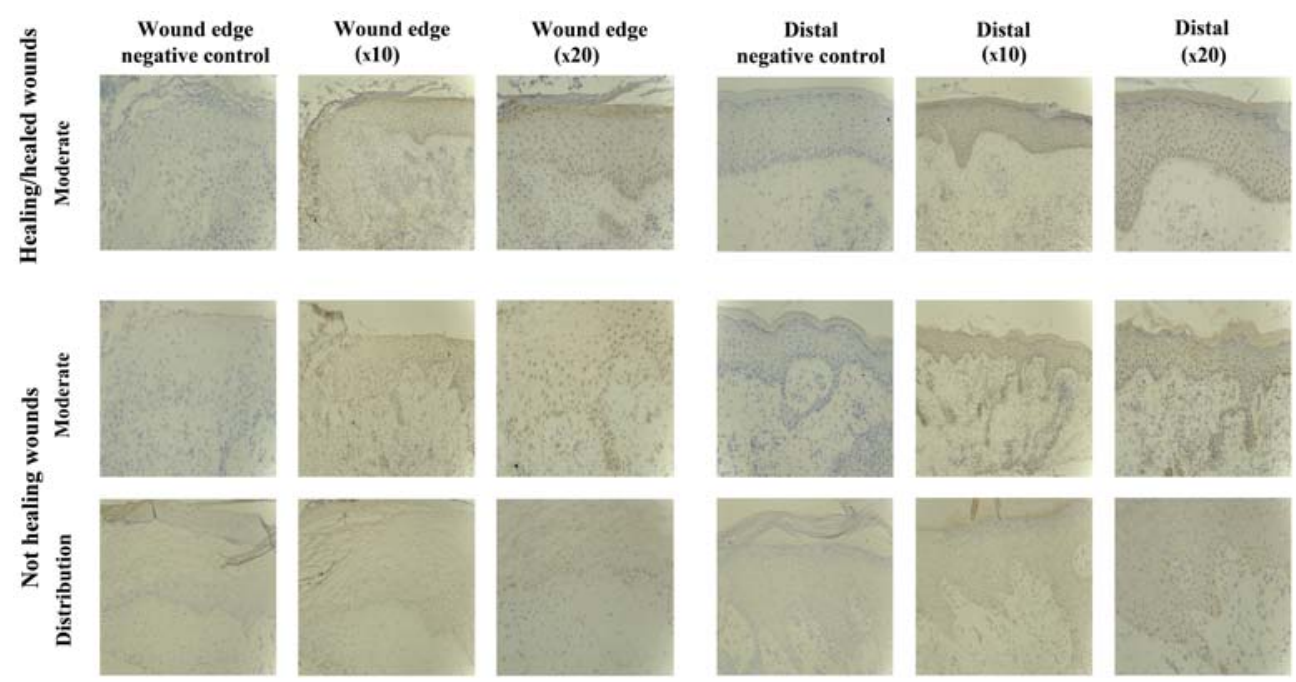

Figure 6. Immunohistochemical (IHC) evaluation of the suppressor of cytokine signalling 5 (SOCS5) protein in healing/healed wounds (top one panel) and non-healing wounds (bottom two panels). The top panel shows representative images of moderate staining detected for SOCS5 in 10 collected healing/healed biopsies. The bottom two panels show representative images from collected non-healing biopsies, demonstrating moderate staining and different protein distribution between wound edge and distal area at two magnifications (x10 and x20) within the sections.

with only two biopsies showing particularly stronger expression of SOCS6 (1/10 healing/healed, 1/10 non-healing (Fig. 7).
Increased SOCS6 expression was identified in the epidermal layers distal to the wound edge in $4 / 20$ biopsies 

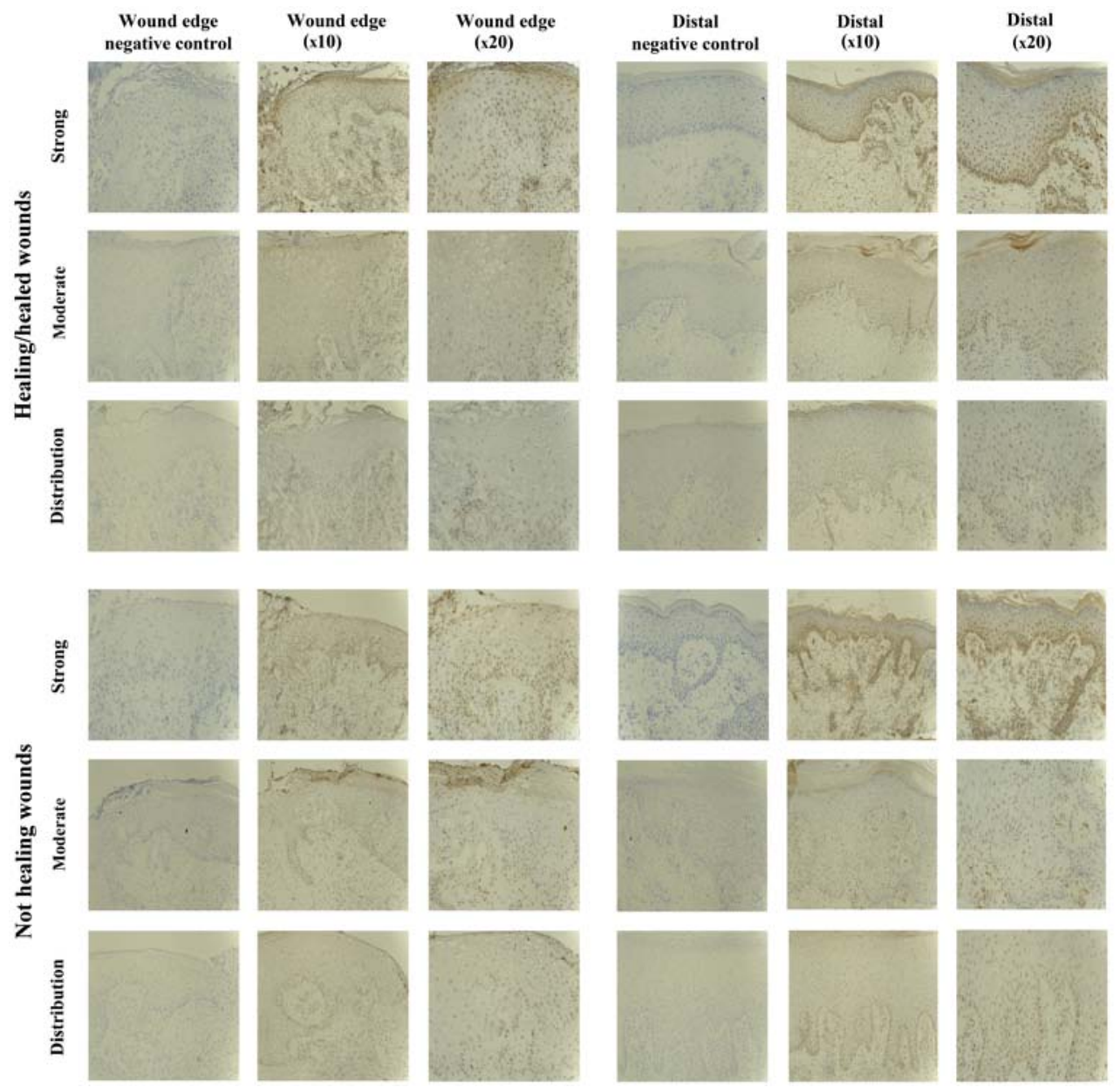

Figure 7. Immunohistochemical (IHC) evaluation of the suppressor of cytokine signalling 6 (SOCS6) protein in healing/healed wounds (top three panels) and non-healing wounds (bottom three panels). Each group shows representative images from the collected biopsies, demonstrating strong staining, moderate staining and different protein distribution between wound edge and distal area at two magnifications (x10 and x20) within the sections.

examined (2/10 healing/healed, 2/10 non-healing), although the remaining biopsies showed uniform staining throughout the tissue samples.

The nucleus of basal and suprabasal keratinocyte cells were particularly positive for SOCS6. However, cytoplasmic staining in other layers was also observed.

SOCS7 staining in chronic tissues. SOCS7 expression was examined throughout the healing/healed and the non-healed chronic wound tissue sections. The sections demonstrated no significant staining profiles when tested with either of the two antibodies available within our laboratories (data not shown). Thus, this molecule appears to demonstrate weak to no expression in the tissues tested in the present study.

\section{Discussion}

Cytokines play important roles in the wound-healing process by attracting inflammatory cells, inducing cell proliferation, differentiation and maturation through various signalling pathways $(2,3)$. Due to these essential roles, some cytokine intervention therapies, such as the usage of platelet-derived growth factor (PDGF) for non-infected foot ulcers, have been licensed and clinically used on diabetic patients (1). However, the mixed presence of cytokines and growth factors at the wound site is concentration- and time-dependent. Therefore, more focused therapies using multiple cytokines and growth factors and/or in a time-specific manner may provide an effective strategy. Such strategies are beginning to be realised and one therapeutic method, termed cytokine gene therapy, has shown the ability to topically deliver strategic cytokines and growth factors at precise time points during the wound-healing process and demonstrates effectiveness in improving the healing of the wounds that have specific genetic defects $(20,21)$. Given the significance of cytokine signalling in wound healing, dysregulation of such signalling may enhance the risk of pathologic and abnormal conditions, potentially leading to delayed healing or non-healing chronic wounds. The JAK-STAT pathway is utilised by most cytokines for signal transduction and is subject to regulation by a number of other molecules including SOCS proteins (5). Therefore, the aim of the present study was to assess the expression pattern of the seven SOCS family members within a clinical cohort of healing and non-healing chronic wounds to examine their potential to act as biomarkers for predicting healing progression.

Our present data, involving the screening of a clinical cohort for the transcript expression of seven SOCS members, show that differences in expression of SOCS family members were observed between the healing/healed and non-healing chronic wounds. Notably, significant differences were obtained for the expression of SOCS3 and 4 between the two 
groups of chronic wounds. In addition, gene level screening performed in the present study suggested that higher, albeit not significant, expression of SOCS1, 2, 5 and 6 in chronic venous leg ulcer patients may indicate poor healing prognosis, whereas a decreased expression of SOCS7 transcript may suggest higher healing potential. However, the low levels of SOCS7 protein expression detected in our study may limit this significance and requires further clarification. Additionally, screening seven members of the SOCS family in chronic wound biopsy sections using IHC analysis did not reveal any obviously distinct differences between the non-healing and healing/healed chronic wounds and in some ways contrasts our transcript analysis. These differences may be due to the smaller subset of samples used in the IHC analysis than in the qPCR analysis. The discrepancy may also have arisen due to the qualitative nature of the IHC study or may be some aspect of post-transcriptional regulation.

SOCS family members have been previously linked to inflammatory disorders and diseases. For example, studies on SOCS1-deficient mouse models have suggested that SOCS1 is an important modulator of interferon- $\gamma($ IFN- $\gamma)$ and allows it to exert protective effects without the risk of hyper-response to viral infection (22). Some of the biopsies in the current study had positive SOCS1 staining in the dermal inflammatory infiltrate. Such results suggested that SOCS1 helps inflammatory cell infiltrate, leading to a prolonged inflammation phase, a major cause of chronic wounds (2). However, dual fluorescent staining may be required to identify whether these positively stained cells were T-lymphocytes, macrophages, fibroblasts or other cells.

SOCS3, acting as an anti-inflammatory regulator, mediates IL-6-gp130 signalling by preventing the activation of STAT3 in macrophages $(23,24)$. Given that chronic inflammatory diseases are promoted by IL-6 and IL-6-related cytokine-mediated STAT3 pathways, and that the expression of SOCS3 is able to inhibit STAT3 activation, it appears that SOCS3 holds the potential to regulate inflammatory diseases related to IL-6 activation (25). Additionally, SOCS3 was found to be a negative regulator of IFN- $\gamma$-induced signalling through the suppression of activated STAT1 (26). In the present study, SOCS3 expression was observed in the cytoplasmic region of mature keratinocytes or the nuclear region in the lower basal and suprabasal layers, particularly distal to the wound edge. SOCS3 expression was also found in inflammatory cells in the dermis below the migratory epidermis and around blood vessels in chronic wound biopsies. This differential SOCS3 expression pattern over different areas of the wound biopsies may indicate a differential regulatory role for SOCS3 in different stages of the wound healing process, although further investigation is required to identify the specific molecule(s) or downstream signalling pathway(s) that may be regulated by SOCS3.

Concerning SOCS5, findings of in vitro studies on the development of helper $\mathrm{T}$ cells suggest that this protein is involved in impairing IL-4-induced STAT6 activation by preferentially interacting with the IL-4 receptor, irrespective of receptor tyrosine phosphorylation. The development of helper T2 (Th2) cells is known to be regulated by cytokine signalling through the IL-4 receptor. Therefore, SOCS5 may act as a negative regulator of $\mathrm{Th} 2$ development by blocking
IL-4 signalling (27). There was only moderate staining for SOCS5 protein in the healing/healed and non-healing chronic wound biopsies compared to that of the other SOCS family members examined in the present study. This result suggests a lower expression pattern of SOCS5 in chronic wound healing relative to the other members. Additionally, SOCS5 staining was mainly observed in the cytoplasmic region in all the layers of the epidemis, which may indicate its regulatory role in keratinocyte behaviour during wound healing.

Furthermore, the proliferation and re-epithelialization phases of wound healing consist of neoangiogenesis, granulation tissue formation, extracellular matrix deposition and re-epithelialization (2). Keratinocytes are important in this stage through the function of migration, proliferation and differentiation. They are defined as major cell components of the epidermis, not only for the function of barrier maintenance, but for its restoration following injury (28). Dysregulation of keratinocyte migratory function is associated with the clinical phenotype of chronic non-healing wounds (29). Direct evidence indicates that SOCS proteins may hold the potential to influence the wound-healing process by regulating the wound site resident cell function. Studies have shown that keratinocyte proliferation and migration are strongly disturbed by the presence of SOCS3, which contributes to impaired wound healing (30), whereas the exacerbated inflammation that characterises chronic wounds is shown to be associated with the overexpression of SOCS3 (31). This evidence may explain the significantly increased expression of SOCS3 transcript levels in the non-healing chronic wound biopsies compared to that in the healing/healed chronic wound biopsies, indicating the upregulation of SOCS3 in non-healing chronic wounds. The IHC staining results on the profile of SOCS3 protein expression, and the distinct proportion (1:4) of the increased SOCS3 protein expression in the epidermis distal to the wound edge throughout 20 chronic wound biospies (1/10 healing/healed, 4/10 non-healing) are noteworthy. They suggest loss of SOCS3 expression on the leading migratory epidermis in the healing/healed chronic wound and/or the relocation of SOCS3 protein between healing/healed and non-healing chronic wounds.

Taken together, SOCS proteins are attractive molecules that may act as novel biomarkers and/or therapeutics to manage chronic wounds, although additional study is required to fully elucidate this role. Thus, establishment of a larger cohort of these tissues, as well as exploring SOCS function and expression across acute and chronic wound tissue and normal skin is required. Additionally, future investigations are required, using in vitro and in vivo models, to determine the potential functional traits of SOCS3 and 4 in representative cell types which play essential roles in the wound-healing process. Such studies may provide additional detail with regard to the precise nature of the SOCS family in the wound-healing process and their true potential to act as novel strategies to combat and manage wound chronicity.

\section{Acknowledgements}

The present study was supported by GlaxoSmithKline and Cancer Research Wales. 


\section{References}

1. Harding KG, Morris HL and Patel GK: Science, medicine and the future: healing chronic wounds. BMJ 324: 160-163, 2002.

2. Behm B, Babilas P, Landthaler M and Schreml S: Cytokines, chemokines and growth factors in wound healing. J Eur Acad Dermatol Venereol 26: 812-820, 2012.

3. Barrientos S, Stojadinovic O, Golinko MS, Brem H and Tomic-Canic M: Growth factors and cytokines in wound healing. Wound Repair Regen 16: 585-601, 2008.

4. Igaz P, Tóth $S$ and Falus A: Biological and clinical significance of the JAK-STAT pathway; lessons from knockout mice. Inflamm Res 50: 435-441, 2001.

5. Rawlings JS, Rosler KM and Harrison DA: The JAK/STAT signaling pathway. J Cell Sci 117: 1281-1283, 2004.

6. Wormald S and Hilton DJ: Inhibitors of cytokine signal transduction. J Biol Chem 279: 821-824, 2004.

7. Starr R, Willson TA, Viney EM, Murray LJ, Rayner JR, Jenkins BJ, Gonda TJ, Alexander WS, Metcalf D, Nicola NA, et al: A family of cytokine-inducible inhibitors of signalling. Nature 387: 917-921, 1997.

8. Endo TA, Masuhara M, Yokouchi M, Suzuki R, Sakamoto H, Mitsui K, Matsumoto A, Tanimura S, Ohtsubo M, Misawa H, et al: A new protein containing an $\mathrm{SH} 2$ domain that inhibits JAK kinases. Nature 387: 921-924, 1997.

9. Naka T, Narazaki M, Hirata M, Matsumoto T, Minamoto S, Aono A, Nishimoto N, Kajita T, Taga T, Yoshizaki K, et al: Structure and function of a new STAT-induced STAT inhibitor. Nature 387: 924-929, 1997.

10. Croker BA, Kiu $\mathrm{H}$ and Nicholson SE: SOCS regulation of the JAK/STAT signalling pathway. Semin Cell Dev Biol 19: 414-422, 2008.

11. Alexander WS: Suppressors of cytokine signalling (SOCS) in the immune system. Nat Rev Immunol 2: 410-416, 2002.

12. Linossi EM, Babon JJ, Hilton DJ and Nicholson SE: Suppression of cytokine signaling: the SOCS perspective. Cytokine Growth Factor Rev 24: 241-248, 2013.

13. Kario E, Marmor MD, Adamsky K, Citri A, Amit I, Amariglio N, Rechavi G and Yarden Y: Suppressors of cytokine signaling 4 and 5 regulate epidermal growth factor receptor signaling. J Biol Chem 280: 7038-7048, 2005 .

14. Krebs DL, Uren RT, Metcalf D, Rakar S, Zhang JG, Starr R, De Souza DP, Hanzinikolas K, Eyles J, Connolly LM, et al: SOCS- 6 binds to insulin receptor substrate 4 , and mice lacking the SOCS-6 gene exhibit mild growth retardation. Mol Cell Biol 22: 4567-4578, 2002.

15. Banks AS, Li J, McKeag L, Hribal ML, Kashiwada M, Accili D and Rothman PB: Deletion of SOCS7 leads to enhanced insulin action and enlarged islets of Langerhans. J Clin Invest 115 2462-2471, 2005.

16. Feng Y, Sanders AJ, Morgan LD, Harding KG and Jiang WG: Potential roles of suppressor of cytokine signaling in wound healing. Regen Med 11: 193-209, 2016.

17. Yasukawa H, Ohishi M, Mori H, Murakami M, Chinen T, Aki D, Hanada T, Takeda K, Akira S, Hoshijima M, et al: IL-6 induces an anti-inflammatory response in the absence of SOCS3 in macrophages. Nat Immunol 4: 551-556, 2003.

18. Bosanquet DC, Harding KG, Ruge F, Sanders AJ and Jiang WG: Expression of IL-24 and IL-24 receptors in human wound tissues and the biological implications of IL-24 on keratinocytes. Wound Repair Regen 20: 896-903, 2012.
19. Parr C, Watkins G, Mansel RE and Jiang WG: The hepatocyte growth factor regulatory factors in human breast cancer. Clin Cancer Res 10: 202-211, 2004.

20. Eriksson E: Gene transfer in wound healing. Adv Skin Wound Care 13 (Suppl 2): 20-22, 2000.

21. Branski LK, Pereira CT, Herndon DN and Jeschke MG: Gene therapy in wound healing: present status and future directions. Gene Ther 14: 1-10, 2007.

22. AlexanderWS,StarrR,FennerJE,ScottCL,HandmanE,Sprigg NS Corbin JE, Cornish AL, Darwiche R, Owczarek CM, et al: SOCS1 is a critical inhibitor of interferon gamma signaling and prevents the potentially fatal neonatal actions of this cytokine. Cell 98: 597-608, 1999.

23. Croker BA, Krebs DL, Zhang JG, Wormald S, Willson TA, Stanley EG, Robb L, Greenhalgh CJ, Förster I, Clausen BE, et al: SOCS3 negatively regulates IL-6 signaling in vivo. Nat Immunol 4: 540-545, 2003.

24. Lang R, Pauleau AL, Parganas E, Takahashi Y, Mages J, Ihle JN, Rutschman R and Murray PJ: SOCS3 regulates the plasticity of gp130 signaling. Nat Immunol 4: 546-550, 2003.

25. Kubo M, Hanada T and Yoshimura A: Suppressors of cytokine signaling and immunity. Nat Immunol 4: 1169-1176, 2003.

26. Song MM and Shuai K: The suppressor of cytokine signaling (SOCS) 1 and SOCS3 but not SOCS2 proteins inhibit interferon-mediated antiviral and antiproliferative activities. J Biol Chem 273: 35056-35062, 1998.

27. Seki Y, Hayashi K, Matsumoto A, Seki N, Tsukada J, Ransom J, Naka T, Kishimoto T, Yoshimura A and Kubo M: Expression of the suppressor of cytokine signaling-5 (SOCS5) negatively regulates IL-4-dependent STAT6 activation and Th2 differentiation. Proc Natl Acad Sci USA 99: 13003-13008, 2002.

28. Pastar I, Stojadinovic O, Yin NC, Ramirez H, Nusbaum AG, Sawaya A, Patel SB, Khalid L, Isseroff RR and Tomic-Canic M: Epithelialization in wound healing: a comprehensive review. Adv Wound Care (New Rochelle) 3: 445-464, 2014.

29. Raja, Sivamani K, Garcia MS and Isseroff RR: Wound re-epithelialization: modulating keratinocyte migration in wound healing. Front Biosci 12: 2849-2868, 2007.

30. Linke A, Goren I, Bösl MR, Pfeilschifter J and Frank S: The suppressor of cytokine signaling (SOCS)-3 determines keratinocyte proliferative and migratory potential during skin repair. J Invest Dermatol 130: 876-885, 2010.

31. Linke A, Goren I, Bösl MR, Pfeilschifter J and Frank S: Epithelial overexpression of SOCS-3 in transgenic mice exacerbates wound inflammation in the presence of elevated TGF-beta1. J Invest Dermatol 130: 866-875, 2010.

This work is licensed under a Creative Commons Attribution 4.0 International (CC BY 4.0) License. 